\title{
LOWNESS FOR THE CLASS OF SCHNORR RANDOM REALS
}

\author{
BJØRN KJOS-HANSSEN, ANDRÉ NIES, AND FRANK STEPHAN
}

\begin{abstract}
We answer a question of Ambos-Spies and Kučera in the affirmative. They asked whether, when a real is low for Schnorr randomness, it is already low for Schnorr tests.

Keywords: lowness, randomness, Schnorr randomness, Turing degrees, recursion theory, computability theory. AMS subject classification 68Q30, 03D25, 03D28.
\end{abstract}

\section{Contents}

1. Introduction

2. Main concepts

2.1. Martingales

2.2. Traceability

3. Statement of the main result

4. Proof of the main result

5. Lowness notions related to Chaitin's halting probability References

\section{INTRODUCTION}

In an influential 1966 paper [9], Martin-Löf proposed an algorithmic formalization of the intuitive notion of randomness for infinite sequences of 0 's and 1's. His formalization was based on an effectivization of a test concept from statistics, by means of uniformly recursively enumerable (r.e.) sequences of open sets. Martin-Löf's proposal addressed some insufficiencies in an earlier algorithmic concept of randomness proposed by Church [3], who had formalized a notion now called computable stochasticity. However, Schnorr [13] criticized Martin-Löf's notion as too strong, because it was based on an r.e. test concept rather than a computable notion of tests.

Department of Mathematics, University of Connecticut, Storrs, CT 06269 (bjorn@math.uconn. edu).

Department of Computer Science, University of Auckland, Private Bag 92019, Auckland, New Zealand (andre@cs.auckland.ac.nz).

School of Computing and Department of Mathematics, National University of Singapore, 3 Science Drive 2, Singapore 117543, Republic of Singapore (fstephan@comp.nus. edu.sg). 
He suggested that one should base a formalization of randomness on computable betting strategies (also called martingales), in a way that would still overcome the problem that Church's concept was too weak. In present terminology, a real $Z$ is computably random if no computable betting strategy succeeds along $Z$; that is, for each computable betting strategy there is a finite upper bound on the capital that it reaches. The real $Z$ is Schnorr random if no martingale succeeds effectively. Here effective success means that the capital at $Z\lceil n$ exceeds $f(n)$ infinitely often, for some unbounded computable function $f$. See [1] for more on the history of these ideas.

We recall some definitions. The Cantor space $2^{\omega}$ is the set of infinite binary sequences; these are called reals and are identified with a set of integers, i.e., subsets of $\omega$. If $\sigma \in 2^{<\omega}$, that is, $\sigma$ is a finite binary sequence, then we denote by $[\sigma]$ the set of reals that extend $\sigma$. These form a basis of clopen sets for the usual discrete topology on $2^{\omega}$. Write $|\sigma|$ for the length of $\sigma \in 2^{<\omega}$. The Lebesgue measure $\mu$ on $2^{\omega}$ is defined by stipulating that $\mu[\sigma]=2^{-|\sigma|}$. With every set $U \subseteq 2^{<\omega}$ we associate the open set $[U] \preceq=$ $\bigcup_{\sigma \in U}[\sigma]$. The empty sequence is denoted $\lambda$. If $\sigma, \tau \in 2^{<\omega}$ and $\sigma$ is a prefix of $\tau$, then we write $\sigma \preceq \tau$. If $\sigma \in 2^{<\omega}$ and $i \in\{0,1\}$, then $\sigma i$ denotes the string of length $|\sigma|+1$ extending $\sigma$ whose final entry is $i$. The concatenation of two strings $\sigma$ and $\tau$ is denoted $\sigma \tau$. The empty set is denoted $\emptyset$, and inclusion of sets is denoted by $\subseteq$. If $A$ is a real and $n \in \omega$, then $A \uparrow n$ is the prefix of $A$ consisting of the first $n$ bits of $A$. Letting $A(n)$ denote bit $n$ of $A$, we have $A\lceil n=A(0) A(1) \cdots A(n-1)$.

Given $\alpha \in 2^{<\omega}$ and a measurable set $C \subseteq 2^{\omega}$, we let $\mu_{\alpha} C=\frac{\mu(C \cap[\alpha])}{\mu[\alpha]}$. For an open set $W$ we let

$$
W \mid \sigma=\bigcup\left\{[\tau]: \tau \in 2^{<\omega},[\sigma \tau] \subseteq W\right\}
$$

Note in particular that $\mu_{\sigma} W=\mu(W \mid \sigma)$ and $\mu_{\lambda} W=\mu W$.

Fixing some effective correspondence between the set of finite subsets of $\omega$ and $\omega$, we let $D_{e}$ be the eth finite subset of $\omega$ under this correspondence. In other words, $e$ is a strong, or canonical, index for the finite set $D_{e}$. Similarly, we let $S_{e}$ be the $e$ th finite subset of $2^{<\omega}$ under a suitable correspondence. Thus $S_{e}$ is a finite set of strings, and $\left[S_{e}\right] \preceq=\cup_{\sigma \in S_{e}}[\sigma]$ is then the clopen set coded by $e \in \omega$. We use the Cantor pairing function, namely the bijection $p: \omega^{2} \rightarrow \omega$ given by $p(n, s)=\frac{(n+s)^{2}+3 n+s}{2}$, and write $\langle n, s\rangle=p(n, s)$.

A Martin-Löf test is a set $U \subseteq \omega \times 2^{\omega}$ such that $\mu U_{n} \leq 2^{-n}$, where $U_{n}$ denotes the $n$th section of $U$, and $U_{n}$ is a $\Sigma_{1}^{0}$ class, uniformly in $n$. If, in addition, $\mu U_{n}$ is a computable real, uniformly in $n$, then $U$ is called a Schnorr test. $Z$ is Martin-Löf random if for each Martin-Löf test $U$ there is an $n$ such that $Z \notin U_{n}$, and Schnorr random if for each Schnorr test $U$ there is an $n$ such that $Z \notin U_{n}$. The notion of Schnorr randomness is unchanged if we instead define a Schnorr test to be a Martin-Löf test for which $\mu U_{n}=2^{-n}$ for each $n \in \omega$. 
Concepts encountered in computability theory are usually based on some notion of computation, and therefore have relativized forms. For instance, we may relativize the tests and randomness notions above to an oracle $A$. If $\mathcal{C}=\{X: X$ is Martin-Löf random $\}$, then the relativization is $\mathcal{C}^{A}=\{X: X$ is Martin-Löf random relative to $A\}$ (meaning that $\Sigma_{1}^{0}$ classes are replaced by $\Sigma_{1}^{0, A}$ classes). In general, if $\mathcal{C}$ is such a relativizable class, we say that $A$ is low for $\mathcal{C}$ if $\mathcal{C}^{A}=\mathcal{C}$. If $\mathcal{C}$ is a randomness notion, more computational power means a smaller class, namely $\mathrm{C}^{A} \subseteq \mathcal{C}$ for any $A$. Being low for $\mathcal{C}$ means having small computational power (in a sense that depends on C). In particular, the low-for- $\mathcal{C}$ reals are closed downward under Turing reducibility.

The randomness notions for which lowness was first considered are MartinLöf and Schnorr randomness. Kučera and Terwijn [8 constructed a noncomputable r.e. set of integers $A$ which is low for Martin-Löf randomness, answering a question of Zambella [16. In the paper [14 it is shown that there are continuously many reals that are low for Schnorr randomness.

An important difference between the two randomness notions is that for Martin-Löf randomness, but not for Schnorr randomness, there is a universal test $R$. Thus, $Z$ is not Martin-Löf random iff $Z \in \bigcap_{b \in \omega} R_{b}$. Therefore, in the Schnorr case, an apparently stronger lowness notion is being low for Schnorr tests, or $S_{0}$-low in the terminology of [1]: $A$ is low for Schnorr tests if for each Schnorr test $U^{A}$ relative to $A$ there is an unrelativized Schnorr test $V$ such that $\bigcap_{n} U_{n}^{A} \subseteq \bigcap_{n} V_{n}$. This implies that $A$ is low for Schnorr randomness, or $S$-low in the terminology of [1. Ambos-Spies and Kučera asked if the two notions coincide. We answer this question in the affirmative.

Terwijn and Zambella [14] actually constructed oracles $A$ that are low for Schnorr tests. They first gave a characterization of this lowness property via a notion of traceability, a restriction on the possible sequence of values of the functions computable from $A$. They showed that $A$ is low for Schnorr tests iff $A$ is computably traceable (see formal definition in the next section). Then they constructed continuously many computably traceable reals. We answer the question of Ambos-Spies and Kučera by showing that each real which is low for Schnorr randomness is in fact computably traceable.

Towards this end, it turns out to be helpful to have a more general view of lowness. We consider lowness for any pair of randomness notions $\mathcal{C}, \mathcal{D}$ with $\mathcal{C} \subseteq \mathcal{D}$.

Definition 1.1. $A$ is in $\operatorname{Low}(\mathcal{C}, \mathcal{D})$ if $\mathcal{C} \subseteq \mathcal{D}^{A}$. We write $\operatorname{Low}(\mathcal{C})$ for Low $(\mathcal{C}, \mathcal{C})$.

Clearly, if $\mathcal{C} \subseteq \widetilde{\mathcal{C}} \subseteq \widetilde{\mathcal{D}} \subseteq \mathcal{D}$ are randomness notions, and the inclusions relativize (so $\widetilde{\mathcal{D}}^{A} \subseteq \mathcal{D}^{A}$ for each real $A$ ), then $\operatorname{Low}(\widetilde{\mathcal{C}}, \widetilde{\mathcal{D}}) \subseteq \operatorname{Low}(\mathcal{C}, \mathcal{D})$. That is, we make the class Low $(\widetilde{\mathcal{C}}, \widetilde{\mathcal{D}})$ larger by decreasing $\mathcal{C}$ or increasing D. Let MR, CR, and SR denote the classes of Martin-Löf random, computably random (defined below), and Schnorr random reals, respectively. Thus, 
for instance, Low $(\mathrm{MR}, \mathrm{CR})$ is the class of oracles $A$ such that each MartinLöf random real is computably random in $A$. We will characterize lowness for any pair of randomness notions $\mathcal{C} \subseteq \mathcal{D}$ with $\mathcal{C}, \mathcal{D} \in\{\mathrm{MR}, \mathrm{CR}, \mathrm{SR}\}$.

Recall that $\Omega$ denotes the halting probability of a universal prefix machine. $\Omega$ is a Martin-Löf random r.e. real, i.e., a real that can be effectively approximated from below. Given $\mathcal{D} \supseteq \mathrm{MR}$, an interesting lowness notion obtained by weakening $\operatorname{Low}(M R, \mathcal{D})$ is $\operatorname{Low}(\{\Omega\}, \mathcal{D})$. That is, instead of $M R \subseteq \mathcal{D}^{A}$ one merely requires that $\Omega \in \mathcal{D}^{A}$. We denote this class by $\operatorname{Low}(\Omega, \mathcal{D})$. In [12], the case $\mathcal{D}=$ MR is studied. The authors show that the class coincides with Low $(\mathrm{MR})$ on the $\Delta_{2}^{0}$ reals but not in general. In fact, a Martin-Löf random real is 2-random iff it is in $\operatorname{Low}(\Omega, M R)$.

Here we investigate the class $\operatorname{Low}(\Omega, \mathrm{SR})$. We show that $A$ is $\operatorname{Low}(\mathrm{MR}$, $\mathrm{SR})$ iff $A$ is r.e. traceable. Moreover, the weaker assumption $\Omega \in \mathrm{SR}^{A}$ still implies that $A$ is array computable (there is a function $f \leq_{w t t} \emptyset^{\prime}$ bounding all functions computable from $A$, on almost all inputs). Thus for r.e. sets of integers $A, A$ being Low(MR, SR) is in fact equivalent to $\Omega \in \mathrm{SR}^{A}$ by Ishmukhametov [5]. We also provide an example of a real $A$ which is array computable but not Low $(\Omega, \mathrm{SR})$.

\section{MAIN CONCEPTS}

2.1. Martingales. For our purposes, a martingale is a function $M: 2^{<\omega} \mapsto$ $\mathbb{Q}$ (where $\mathbb{Q}$ is the set of rational numbers) such that (i) the domain of $M$ is $2^{<\omega}$, or $2^{\leq n}=\left\{\sigma \in 2^{<\omega}:|\sigma| \leq n\right\}$ for some $n$, (ii) $M(\lambda) \leq 1$, and (iii) $M$ has the martingale property $M(x 0)+M(x 1)=2 M(x)$ whenever the strings $x 0, x 1$ belong to the domain of $M$. A martingale $M$ succeeds on a sequence $Z \in 2^{\omega}$ if

$$
\limsup _{n \rightarrow \infty} M(Z\lceil n)=\infty
$$

A real is computably random if no computable martingale succeeds on it.

A martingale $M$ effectively succeeds on a sequence $Z$ if there is a nondecreasing and unbounded computable function $h: \omega \longrightarrow \omega$ such that

$$
\limsup _{n \rightarrow \infty} M(Z\lceil n)-h(n)>0 .
$$

Equivalently (since we are considering integer-valued functions), $\exists^{\infty} n M(Z \uparrow$ $n)>h(n)$. We can now state the characterization of Schnorr randomness in terms of martingales: a real $Z$ is Schnorr random iff no computable martingale effectively succeeds on $Z$.

2.2. Traceability. Let $W_{e}$ denote the eth r.e. set of integers in some standard list. A real $A$ is r.e. traceable if there is a computable function $p$, called a bound, such that for every $f \leq_{T} A$ there is a computable function $r$ such that for all $x$ we have $\left|W_{r(x)}\right| \leq p(x)$ and $f(x) \in W_{r(x)}$.

The following is a stronger notion than r.e. traceability. $A$ is computably traceable if there is a computable $p$ such that for every $f \leq_{T} A$ there is a computable $r$ such that for all $x$ we have $\left|D_{r(x)}\right| \leq p(x)$ and $f(x) \in D_{r(x)}$. 
It is interesting to notice that it does not matter what bound $p$ one chooses as a witness for traceability; see the following.

Proposition 2.1 (see Terwijn and Zambella [14]). Let $A$ be a real that is computably traceable with bound $p$. Then for any monotone and unbounded computable function $p^{\prime}, A$ is computably traceable with bound $p^{\prime}$. The same holds for r.e. traceability.

The result of Terwijn and Zambella is the following.

Theorem 2.2 (see [14]). A real $A$ is low for Schnorr tests iff $A$ is computably traceable.

\section{Statement of the main Result}

\section{Theorem 3.1.}

(I) $A$ is $\operatorname{Low}(\mathrm{MR}, \mathrm{SR})$ iff $A$ is r.e. traceable.

(II) $A$ is $\operatorname{Low}(\mathrm{CR}, \mathrm{SR})$ iff $A$ is $\operatorname{Low}(\mathrm{SR})$ iff $A$ is computably traceable.

We make some remarks about the proofs and fill in the details in the next section. We obtain Theorem 3.1. (I) by modifying the methods in [14 to the case of r.e. traces instead of computable ones.

As for Theorem 3.1(II), by Theorem 2.2 if $A$ is computably traceable, then $A$ is low for Schnorr tests. Hence $A$ is certainly Low(SR), and therefore also Low $(\mathrm{CR}, \mathrm{SR})$. It remains only to show that each real $A \in \operatorname{Low}(\mathrm{CR}, \mathrm{SR})$ is computably traceable. To see that this is so, take the following three steps:

1. Recall that $A$ is hyperimmune-free if for each $g \leq_{T} A$ there is a computable $f$ such that for all $x$ we have $g(x) \leq f(x)$. As a first step towards proving Theorem 3.1(II), Bedregal and Nies [2] showed that each $A \in \operatorname{Low}(\mathrm{CR}, \mathrm{SR})$ is hyperimmune-free (see Lemma 4.9 below). To see this, assume that $A$ is not, so there is a function $g \leq_{T} A$ not dominated by any computable function $f$. Define a martingale $L \leq_{T} A$ which succeeds in the sense of Schnorr, with the computable lower bound $h(n)=n / 4$, on some $Z \in \mathrm{CR}$. One uses here that $g$ is infinitely often above the running time of each computable martingale. (Special care has to be taken with the partial martingales, which results in a real $Z$ that is only $\Delta_{3}^{0}$.)

2. If $A$ is hyperimmune-free and r.e. traceable, then $A$ is computably traceable. If we let $g \leq_{T} A$, then the first stage where $g(x)$ appears in a given trace for $g$ can be computed relative to $A$.

3. Now each $A$ in $\operatorname{Low}(\mathrm{CR}, \mathrm{SR})$ is r.e. traceable by Theorem 3.1(I), and hence by the above is computably traceable, and Theorem 3.1(II) follows.

We discuss lowness for the remaining pairs of randomness notions. Nies has shown that $A$ is $\operatorname{Low}(\mathrm{MR}, \mathrm{CR})$ iff $A$ is Low(MR) iff $A$ is $K$-trivial, where $A$ is $K$-trivial if for all $n K(X\lceil n) \leq K(n)+O(1)$ (see [11]). Here $K(\sigma)$ denotes the prefix-free Kolmogorov complexity of $\sigma \in 2^{<\omega}$. Finally, he shows that a real $A$ which is Low(CR) is computable; namely, $A$ is both 
$K$-trivial and hyperimmune-free. Since all $K$-trivial reals are $\Delta_{2}^{0}$, and all hyperimmune-free $\Delta_{2}^{0}$ reals are computable, the conclusion follows.

\section{Proof of the main Result}

We first need to develop a few useful facts from measure theory.

Definition 4.1. A measurable set $A$ has density $d$ at a real $X$ if

$$
\lim _{n \rightarrow \infty} \mu_{(X\lceil n)} A=d
$$

A basic result is the following.

Theorem 4.2 (Lebesgue density theorem). Let $\Xi(A)=\{X: A$ has density 1 at $X\}$. If $A$ is a measurable set, then so is $\Xi(A)$, and the measure of the symmetric difference of $A$ and $\Xi(A)$ is zero.

Corollary 4.3. Let $C$ be a measurable subset of $2^{\omega}$, with $\mu C>0$. Then for each $\delta<1$ there is an $\alpha \in 2^{<\omega}$ such that $\mu_{\alpha} C \geq \delta$.

We will use the following consequence of Corollary 4.3.

Lemma 4.4. Let $0<\epsilon \leq 1$. If $U_{n}, n \in \omega$, and $V$ are open subsets of $2^{\omega}$ with $\bigcap_{n \in \omega} U_{n} \subseteq V$ and $\mu V<\epsilon$, then there exist $\sigma$ and $n$ such that $\mu_{\sigma}\left(U_{n}-V\right)=0$ and $\mu_{\sigma} V<\epsilon$.

Proof. Suppose otherwise; we shall obtain a contradiction by constructing a real in $\bigcap_{n \in \omega} U_{n}-V$. Let $\sigma_{0}=\lambda$ and assume we have defined $\sigma_{n}$ such that $\mu_{\sigma_{n}} V<\epsilon$. By hypothesis, $\mu_{\sigma_{n}}\left(U_{n}-V\right)>0$, and thus there is a $[\tau] \subseteq U_{n}$ such that $\mu_{\sigma_{n}}([\tau]-V)>0$. In particular, $\tau \succeq \sigma_{n}$ and $\mu_{\tau} V<1$. Let $C=2^{\omega}-V$, a closed and hence measurable set. By Corollary 4.3 applied to $C$ (and with $2^{\omega}$ replaced by $[\tau]$ ), there exists $\sigma_{n+1} \succeq \tau$ such that $\mu_{\sigma_{n+1}} V<\epsilon$. Let $X$ be the real that extends all $\sigma_{n}$ 's constructed in this way. Since $\left[\sigma_{n+1}\right] \subseteq U_{n}$ for all $n$, we have that $X \in \bigcap_{n \in \omega} U_{n}$. However, $\left[\sigma_{n}\right] \nsubseteq V$ for every $n$, so, since $V$ is open, $X \notin V$. This contradiction completes the proof.

We now get to the proof of Theorem 3.1. First we show Theorem 3.1(I), namely, that $A$ is $\operatorname{Low}(\mathrm{MR}, \mathrm{SR})$ iff $A$ is r.e. traceable. We start with the "६" direction.

Lemma 4.5. If $A$ is r.e. traceable, then $A$ is $\operatorname{Low}(\mathrm{MR}, \mathrm{SR})$.

Proof. Assume that $A$ is r.e. traceable and that $U^{A}$ is a Schnorr test relative to $A$. Let $U_{n, s}^{A}, n, s \in \omega$, be clopen sets, $U_{n, s}^{A} \subseteq U_{n, s+1}^{A}, U_{n}^{A}=\bigcup_{s \in \omega} U_{n, s}^{A}$, such that the $U_{n, s}^{A}$ are $\Delta_{1}^{0, A}$ classes uniformly in $n$ and $s$. As $\mu U_{n}^{A}=2^{-n}$, we may assume that $\mu U_{n, s}^{A}>2^{-n}\left(1-2^{-s}\right)$. Let $f$ be an $A$-computable function such that $\left[S_{f(\langle n, s\rangle)}\right]=U_{n, s}^{A}$. Since $A$ is r.e. traceable and $f \leq_{T} A$, we can let $T$ be an r.e. trace of $f$. By Proposition 2.1, we may choose $T$ such that in addition $\left|T_{x}\right| \leq x$ for each $x>0$. 
We now want to define a subtrace $\hat{T}$ of $T$, i.e., $\hat{T}_{\langle n, s\rangle} \subseteq T_{\langle n, s\rangle}$ for each $n, s$. The intent is that the open sets defined via $\hat{T}$ are small enough to give us a Martin-Löf test containing $\cap_{n \in \omega} U_{n}^{A}$, and nothing important is in $T_{\langle n, s\rangle}-\hat{T}_{\langle n, s\rangle}$. Thus let $\hat{T}_{\langle n, s\rangle}$ be the set of $e \in T_{\langle n, s\rangle}$ such that $2^{-n}\left(1-2^{-s}\right) \leq$ $\mu\left[S_{e}\right] \preceq \leq 2^{-n}$ and $\left[S_{e}\right] \preceq \supseteq\left[S_{d}\right] \preceq$ for some $d \in \hat{T}_{\langle n, s-1\rangle}$, where $\hat{T}_{\langle n,-1\rangle}=\omega$. Let

$$
V_{n}=\bigcup\left\{\left[S_{e}\right] \preceq: e \in \hat{T}_{\langle n, s\rangle}, s \in \omega\right\} .
$$

Then $\mu V_{n} \leq 2^{-n}\left|\hat{T}_{\langle n, 0\rangle}\right|+\sum_{s \in \omega} 2^{-s} 2^{-n}\left|\hat{T}_{\langle n, s\rangle}\right|$. Since $\left|\hat{T}_{\langle n, s\rangle}\right| \leq\left|T_{\langle n, s\rangle}\right| \leq$ $\langle n, s\rangle$ for $\langle n, s\rangle \neq 0$, and $\langle n, s\rangle$ has only polynomial growth in $n$ and $s$, it is clear that $\sum_{s \in \omega} 2^{-s} 2^{-n}\left|\hat{T}_{\langle n, s\rangle}\right|$ is finite and goes effectively to 0 as $n \rightarrow \infty$; hence the same can be said of $\mu V_{n}$. Thus there is a recursive function $f$ such that $\mu V_{f(n)} \leq 2^{-n}$. Let $\tilde{V}_{n}=V_{f(n)}$. Then $\tilde{V}$ is a Martin-Löf test and $\bigcap_{n} U_{n}^{A} \subseteq \bigcap_{n} \tilde{V}_{n}$. That is, each Schnorr test relative to $A$ is contained in a Martin-Löf test. It follows that each real that is Martin-Löf random is Schnorr random relative to $A$, and the proof is complete.

Next we will show the " $\Rightarrow$ " direction of Theorem 3.1(I). The proof is similar to the " $\Rightarrow$ " of Theorem 2.2 .

Definition 4.6. For $k, l \in \omega$ define the clopen set

$$
B_{k, l}=\bigcup\left\{\left[\tau 1^{k}\right]: \tau \in 2^{<\omega},|\tau|=l\right\},
$$

where $1^{k}$ is a string of 1 's of length $k$.

Note that $\mu B_{k, l}=2^{-k}$ for all $l$.

Lemma 4.7. If $A \in 2^{\omega}$ is $\operatorname{Low}(\mathrm{MR}, \mathrm{SR})$, then $A$ is r.e. traceable.

Proof. Note that $A$ is $\operatorname{Low}(\mathrm{MR}, \mathrm{SR})$ iff for every Schnorr test $U^{A}$ relative to $A, \bigcap_{n \in \omega} U_{n}^{A} \subseteq \bigcap_{b \in \omega} R_{b}$ (recall that $R$ is a universal Martin-Löf test).

Oversimplifying a bit, one can say that the proof below goes as follows. We code a given $g \leq_{T} A$ into a Schnorr test $U^{g}$ relative to $A$. Then, by hypothesis, $\bigcap_{n} U_{n}^{g} \subseteq \bigcap_{n} R_{n}$; in fact we will use only the fact that $\bigcap_{n} U_{n}^{g} \subseteq$ $R_{3}$. We then define an r.e.trace $T$; namely, $T_{k}$ is the set of $l$ such that $B_{k, l}-R_{3}$ has small measure in some sense. Since $R_{3}$ has rather small measure, $B_{k, l}-R_{3}$ will tend to have big measure, which means that there will be only a few $l$ for which $B_{k, l}-R_{3}$ has small measure; in other words, $T_{k}$ has small size. Moreover, we make sure $T$ is a trace for $g$ so that $A$ is r.e. traceable.

We now give the proof details. Suppose that we want to find a trace for a given function $g \leq_{T} A$. We define the test $U^{g}$ by stipulating that

$$
U_{n}^{g}=\bigcup_{k>n} B_{k, g(k)}
$$

It is easy to see that $\mu U_{n}^{g}$ can be approximated computably in $A$, so after taking a subsequence of $U_{n}^{g}, n \in \omega$, we may assume that $U^{g}$ is a Schnorr 
test relative to $A$. Hence, by assumption, $\bigcap U^{g} \subseteq \bigcap_{b \in \omega} R_{b}$. Thus $V=R_{3}$ contains $\bigcap U^{g}$ and $\mu V<\frac{1}{4}$. We may assume throughout that $g(k) \geq k$ for every $k$ because from a trace for $g(k)+k$ one can obtain a trace for $g$ with the same bound. By Lemma 4.4, there exist $\sigma$ and $n$ such that $\mu_{\sigma}\left(U_{n}^{g}-V\right)=0$ and $\mu_{\sigma} V<1 / 4$. As $U_{0}^{g} \supseteq U_{1}^{g} \supseteq \cdots$, we can choose $\sigma$ and $n$ with the additional property $n \geq|\sigma|$. Hence for each $k>n$, we have $g(k) \geq k>n \geq|\sigma|$ and hence $g(k) \geq|\sigma|$.

Let $\tilde{V}=V \mid \sigma$, let $\tilde{g}(k)=\max \{0, g(k)-|\sigma|\}$, and take

$$
T_{k}=\left\{l: \mu\left(B_{k, l}-\tilde{V}\right)<2^{-(l+3)}\right\} .
$$

Note that for each $l \in \omega$, if $l \geq|\sigma|$, then $B_{k, l} \mid \sigma=B_{k, l-|\sigma|}$. Thus, since $g(k) \geq|\sigma|$

$$
U_{n}^{g}\left|\sigma=\bigcup_{k>n} B_{k, g(k)}\right| \sigma=\bigcup_{k>n} B_{k, g(k)-|\sigma|}=U_{n}^{\tilde{g}},
$$

and so $\mu\left(U_{n}^{\tilde{g}}-\tilde{V}\right)=\mu_{\sigma}\left(U_{n}^{g}-V\right)=0$. Hence $\tilde{g}(k) \in T_{k}$ for all $k>n$.

Since $\tilde{V}$ is a $\Sigma_{1}^{0}$ class, it is evident that $T$ is an r.e. set of integers; indeed $B_{k, l}-\tilde{V}$ is a $\Pi_{1}^{0}$ class, and thus we can enumerate the fact that certain basic open sets $[\sigma]$ are disjoint from it, until the measure remaining is as small as required. A trace for $g$ is obtained as follows:

$$
G_{k}= \begin{cases}\left\{l+|\sigma|: l \in T_{k}\right\} & \text { if } k>n, \\ \{g(k)\} & \text { if } k \leq n .\end{cases}
$$

We now show that $G$ is a trace for $g$; i.e., for all $k \in \omega, g(k) \in G_{k}$. If $k \leq n$, then this holds by definition of $G_{k}$; thus suppose $k>n$. Then $g(k)>k>n>|\sigma|$, so $\tilde{g}(k)=g(k)-|\sigma|$, so $g(k)=\tilde{g}(k)+|\sigma|$. As $k>n$, $\tilde{g}(k) \in T_{k}$ and hence $g(k) \in G_{k}$.

Clearly $G$ is r.e.; thus it remains to show that $\left|G_{k}\right|$ is computably bounded, independently of $g$. As $\left|G_{k}\right|=\left|T_{k}\right|$ for $k>n$ and $\left|G_{k}\right|=1$ for $k \leq n$, this is a consequence of Lemma 4.8 below.

Lemma 4.8. If $\tilde{V}$ is a measurable set with $\mu \tilde{V}<\frac{1}{4}$, and $T_{k}=\left\{l: \mu\left(B_{k, l}-\right.\right.$ $\left.\tilde{V})<2^{-(l+3)}\right\}$, then for $k \geq 1,\left|T_{k}\right|<2^{k} k$.

Proof. Observe that, by definition of $T_{k}$,

$$
\sum_{l \in T_{k}} \mu\left(B_{k, l}-\tilde{V}\right)<\sum_{l \in T_{k}} 2^{-(l+3)} \leq \frac{1}{8} \sum_{l \in \omega} 2^{-l}=\frac{1}{4},
$$

so

$$
\mu \bigcup_{l \in T_{k}} B_{k, l}-\mu \tilde{V} \leq \mu \bigcup_{l \in T_{k}}\left(B_{k, l}-\tilde{V}\right) \leq \frac{1}{4} .
$$

As $\mu \tilde{V}<\frac{1}{4}$, we obtain that

$$
\mu \bigcup_{l \in T_{k}} B_{k, l}<\frac{1}{2}
$$


As observed above, $\mu B_{k, l}=2^{-k}$. Moreover, for $k$ fixed, the $B_{k, l}$ 's are mutually independent as soon as the l's are taken sufficiently far apart. In fact, sufficiently far here means a distance of $k$. So for $k \geq 1$ we let $T_{k}^{*}$ be a subset of $T_{k}$ consisting of $\left\lfloor\frac{\left.\mid T_{k}\right\rfloor}{k}\right\rfloor$ elements, all of which are sufficiently far apart. (Here $\lfloor a\rfloor$ is the greatest integer $\leq a$.) To show that such a set exists we may assume we are in the worst case, where the elements of $T_{k}$ are closest together: say, $T_{k}=\left\{0, \ldots,\left|T_{k}\right|-1\right\}$. Then let $T_{k}^{*}=\{m k: 0 \leq m \leq$ $\left.\left\lfloor\frac{\left.\mid T_{k}\right\rfloor}{k}\right\rfloor-1\right\}$. As $\left(\left\lfloor\frac{\left|T_{k}\right|}{k}\right\rfloor-1\right) k \leq\left|T_{k}\right|-k \leq\left|T_{k}\right|-1 \in T_{k}$, this makes $T_{k}^{*} \subseteq T_{k}$. Write $\alpha=\left\lfloor\frac{\left|T_{k}\right|}{k}\right\rfloor$. We now have

$$
\mu \bigcap_{l \in T_{k}}\left(2^{\omega}-B_{k, l}\right) \leq \mu \bigcap_{l \in T_{k}^{*}}\left(2^{\omega}-B_{k, l}\right)=\left(1-2^{-k}\right)^{\alpha}
$$

and hence

$$
\begin{gathered}
1-\left(1-2^{-k}\right)^{\alpha} \leq 1-\mu \bigcap_{l \in T_{k}}\left(2^{\omega}-B_{k, l}\right)=\mu 2^{\omega}-\mu \bigcap_{l \in T_{k}}\left(2^{\omega}-B_{k, l}\right) \\
\leq \mu\left(2^{\omega}-\bigcap_{l \in T_{k}}\left(2^{\omega}-B_{k, l}\right)\right)=\mu \bigcup_{l \in T_{k}} B_{k, l}<\frac{1}{2} .
\end{gathered}
$$

From the inequality above we obtain, letting $m=2^{k}-1$,

$$
\left(1-\frac{1}{m+1}\right)^{\alpha}=\left(1-2^{-k}\right)^{\alpha}>\frac{1}{2}
$$

or $\left(\frac{m+1}{m}\right)^{\alpha}<2$. Now suppose $\alpha \geq m$. Then $\left(\frac{m+1}{m}\right)^{\alpha} \geq\left(\frac{m+1}{m}\right)^{m} \geq 2$ as $(m+1)^{m} \geq m^{m}+m^{m-1}\left(\begin{array}{c}m \\ 1\end{array}\right)=2 m^{m}$. Thus we conclude $\alpha<m=2^{k}-1$. Now, by the definition of $\alpha$, we have $\frac{T_{k}}{k} \leq \alpha+1<2^{k}$ and so $\left|T_{k}\right|<2^{k} k$; this completes the proof.

In order to prove Theorem 3.1(II), recall that, by Theorem 2.2, each computably traceable real is Low $(\mathrm{SR})$. Thus it suffices to show that each Low $(\mathrm{CR}, \mathrm{SR})$ real is computably traceable. The first ingredient for showing this is the following result from [2].

Lemma 4.9. If $A$ is $\operatorname{Low}(\mathrm{CR}, \mathrm{SR})$, then $A$ is hyperimmune-free.

Proof. Suppose $A$ is not hyperimmune-free, so that there is a function $g \leq_{T} A$ not dominated by any computable function. Thus, for each computable $f, \exists^{\infty} x f(x) \leq g(x)$. We will define a computably random real $X$ and an $A$-computable martingale $L$ that succeeds on $X$ in the sense of Schnorr, so that $A$ is not Low (CR, SR). In the following, $\alpha, \beta, \gamma$ denote finite subsets of $\omega$, and $n_{\alpha}=\sum_{i \in \alpha} 2^{i}$ (here $n_{\emptyset}=0$ ).

Let $\left\{M_{e}\right\}_{e \in \omega}$ be an effective listing of all partial computable martingales with range included in $[1 / 2, \infty)$. At stage $t$, we have a finite portion $M_{e}[t]$, whose domain is a subset of some set of the form $2^{\leq n}$ for some $n$. If $X$ is 
not computably random, then $\lim _{n \rightarrow \infty} M_{e}\left(X\lceil n)=\infty\right.$ for some total $M_{e}$ by [13. Let

$$
T M G=\left\{e: M_{e} \text { is total }\right\} .
$$

For finite sets $\alpha, \beta$, let us in this proof say that $\alpha$ is a strong subset of $\beta$ (denoted $\alpha \subseteq^{+} \beta$ ) if $\alpha \subseteq \beta$ and moreover for each $i \in \omega$, if $i \in \beta-\alpha$, then $i>\max (\alpha)$. Thus the possibility that $\beta$ contains an element smaller than some element of $\alpha$ is ruled out.

For certain $\alpha$, and all those included in $T M G$, we will define strings $x_{\alpha}$ in such a way that $\alpha \subseteq^{+} \beta \Rightarrow x_{\alpha} \preceq x_{\beta}$. We choose the strings in such a way that $M_{e}\left(x_{\alpha}\right)$ is bounded by a fixed constant (depending on $e$ ) for each total $M_{e}$ and each $\alpha$ containing $e$. Then the set of integers

$$
X=\bigcup_{e \in \omega} x_{T M G \cap[0, e]}
$$

is a computably random real. On the other hand, we are able to define an $A$-computable martingale $L$ which Schnorr succeeds on $X$. We give an inductive definition of the strings $x_{\alpha}$, "scaling factors" $p_{\alpha} \in \mathbb{Q}^{+}$(positive rationals) (we do not define $p_{\emptyset}$ ), and partial computable martingales $M_{\alpha}$ such that if $x_{\alpha}$ is defined, then

$$
M_{\alpha}\left(x_{\alpha}\right) \text { converges in } g\left(\left|x_{\alpha}\right|\right) \text { steps and } M_{\alpha}\left(x_{\alpha}\right)<2 .
$$

It will be clear that $A$ can decide if $y=x_{\alpha}$, given inputs $y$ and $\alpha$.

Let $x_{\emptyset}=\lambda$, and let $M_{\emptyset}$ be the constant zero function. (We may assume that $g$ is such that $M_{\emptyset}(\lambda)$ converges in $g(0)$ steps.) Now suppose $\alpha=\beta \cup\{e\}$, where $e>\max (\beta)$, and inductively suppose that (11) holds for $\beta$. Let

$$
p_{\alpha}=\frac{1}{2} 2^{-\left|x_{\beta}\right|}\left(2-M_{\beta}\left(x_{\beta}\right)\right),
$$

and let $M_{\alpha}=M_{\beta}+p_{\alpha} M_{e}$. Since $M_{e}$ is a martingale on its domain, $M_{e}(z) \leq$ $2^{|z|}$ for any $z$. So, writing $b=M_{\beta}\left(x_{\beta}\right)$, we have $M_{\alpha}\left(x_{\beta}\right)=b+p_{\alpha} M_{e}\left(x_{\beta}\right)<$ $b+p_{\alpha} 2^{\left|x_{\beta}\right|}=b+\frac{1}{2}(2-b)=1+\frac{b}{2}<1+\frac{2}{2}=2$ if $M_{\alpha}\left(x_{\beta}\right)$ is defined.

To define $x_{\alpha}$, we look for a sufficiently long $x \succeq x_{\beta}$ such that $M_{\alpha}$ does not increase from $x_{\beta}$ to $x$ and $M_{\alpha}(x)$ converges in $g(|x|)$ steps. In detail, for larger and larger $m>\left|x_{\beta}\right|, m \geq 4 n_{\alpha}$, if no string $y,|y|<m$, has been designated to be $x_{\alpha}$ as yet, and if $M_{\alpha}(z)$ (i.e., each $\left.M_{e}(z), e \in \alpha\right)$ converges in $g(m)$ steps, for each string $z$ of length $\leq m$, then choose $x_{\alpha}$ of length $m$, $x_{\beta} \prec x_{\alpha}$ such that $M_{\alpha}$ does not increase anywhere from $x_{\beta}$ to $x_{\alpha}$.

Claim 4.10. If $\alpha \subseteq T M G$, then $x_{\alpha}$ and $p_{\alpha}$ (the latter only if $\alpha \neq \emptyset$ ) are defined.

Proof. The claim is trivial for $\alpha=\emptyset$. Suppose that it holds for $\beta$, and $\alpha=\beta \cup\{e\} \subseteq T M G$, where $e>\max (\beta)$. Since the function

$$
f(m)=\mu s \quad \forall e \in \alpha, \forall x \quad\left[|x| \leq m \Rightarrow M_{e}(x) \text { converges in } s \text { steps }\right]
$$


is computable, there is a least $m \geq 4 n_{\alpha}, m>\left|x_{\beta}\right|$ such that $g(m) \geq f(m)$. Since there is a path down the tree starting at $x_{\beta}$, where $M_{\alpha}$ does not increase, the choice of $x_{\alpha}$ can be made.

Claim 4.11. If $\beta \subseteq \subseteq^{+} \alpha$ are finite sets, then $M_{\beta}(x) \leq M_{\alpha}(x)$ for all $x$.

Proof. This is clear by induction from the case $\alpha=\beta \cup\{e\}$, i.e., the case where $\alpha-\beta$ has only one element.

Claim 4.12. $X$ is computably random.

Proof. Suppose that $M_{e}$ is total. Let $\alpha=T M G \cap[0, e]$. Suppose $\alpha \subseteq \gamma$, $\gamma^{\prime}=\gamma \cup\{i\}, \max (\gamma)<i$, and $\gamma^{\prime} \subseteq T M G$. Then $\alpha \subseteq^{+} \gamma \subseteq^{+} \gamma^{\prime}$. Hence by Claim 4.11, for each $x$ with $x_{\gamma} \preceq x \preceq x_{\gamma^{\prime}}$, we have

$$
p_{\alpha} M_{e}(x) \leq M_{\alpha}(x) \leq M_{\gamma^{\prime}}(x) \leq M_{\gamma^{\prime}}\left(x_{\gamma}\right)<2,
$$

and hence $M_{e}(x)<2 / p_{\alpha}$ for each $x \prec X$, and so the capital of $M_{e}$ on $X$ is bounded.

Claim 4.13. There is a martingale $L \leq_{T} A$ which effectively succeeds on $X$. In fact,

$$
\exists^{\infty} x \prec X L(x) \geq\left\lfloor\frac{|x|}{4}\right\rfloor .
$$

Proof. For a string $z$, let $r(z)=\lfloor|z| / 2\rfloor$. We let $L=\sum_{\alpha} L_{\alpha}$, where $L_{\alpha}$ is a martingale with initial capital $L_{\alpha}(\lambda)=2^{-n_{\alpha}}$, which bets everything along $x_{\alpha}$ from $x_{\alpha} \uparrow r\left(x_{\alpha}\right)$ on. More precisely, if $x_{\alpha}$ is undefined, then $L_{\alpha}$ is constant with value $2^{-n_{\alpha}}$. Otherwise, for convenience we let $x=x_{\alpha}\left\lceil 2 r\left(x_{\alpha}\right)\right.$ and work with $x$ instead of $x_{\alpha}$; we define $L_{\alpha}$ on a string $y$ as follows:

- If $y$ does not contain "half of $x$," i.e., if $x \uparrow r(x) \npreceq y$, then just let $L_{\alpha}(y)=2^{-n_{\alpha}}$.

- If $y$ does contain "half of $x$ " but $y$ and $x$ are incompatible, then let $L_{\alpha}(y)=0$.

- If $y$ contains "half of $x$ " and $x$ and $y$ are compatible, then let $L_{\alpha}(y)=$ $2^{-n_{\alpha}} 2^{\min (|y|-r(x), r(x))}$.

Thus if $y$ contains $x$, then $L_{\alpha}(y)=2^{r(x)-n_{\alpha}}$, so we make no more bets once we extend $x_{\alpha}$, and if $x$ contains $y$, then $L_{\alpha}(y)=2^{|y|-r(x)-n_{\alpha}}$; i.e., we double the capital for each correct bit of $x$ beyond $x\lceil r(x)$.

Note that $L(\lambda)=\sum_{\alpha} 2^{-n_{\alpha}}$, and, as each $k \in \omega$ has a unique binary expansion and hence is equal to $n_{\alpha}$ for a unique finite set $\alpha$, we have $L(\lambda)=$ $\sum_{k \in \omega} 2^{-k}=2$. Moreover, it is clear that each $L_{\alpha}$ satisfies the martingale property $L_{\alpha}(x 0)+L_{\alpha}(x 1)=2 L_{\alpha}(x)$, and hence so does $L$.

$L$ effectively succeeds on $X$. Indeed, as $\left|x_{\alpha}\right| \geq 4 n_{\alpha}$, we have $L_{\alpha}\left(x_{\alpha}\right)=$ $2^{r\left(x_{\alpha}\right)-n_{\alpha}} \geq 2^{\left\lfloor\left|x_{\alpha}\right| / 2\right\rfloor-\left\lfloor\left|x_{\alpha}\right| / 4 \mid\right\rfloor} \geq 2^{\left\lfloor\left|x_{\alpha}\right| / 4\right\rfloor} \geq\left\lfloor\left|x_{\alpha}\right| / 4\right\rfloor$ since $2^{q} \geq q$ for each $q \in \omega$. 
Finally, we show that $L \leq_{T} A$. Given input $y$, we use $g$ to see if some string $x,|x| \leq 2|y|$, is $x_{\alpha}$. If not, $L_{\alpha}(y)=2^{-n_{\alpha}}$. Else we determine $L_{\alpha}(y)$ from $x$ using the definition of $L_{\alpha}$.

The second ingredient to the proof of Theorem 3.1(II) is the following fact of independent interest.

Proposition 4.14. If $A$ is hyperimmune-free and r.e. traceable, then $A$ is computably traceable.

Proof. Let $f \leq_{T} A$, and let $h$ be as in the definition of r.e. traceability. Let $g(x)=\mu s\left(f(x) \in W_{h(x), s}\right.$ ) (where $W_{e, s}$ is the approximation at stage $s$ to the r.e. set $\left.W_{e}\right)$. Then $g \leq_{T} A$, and so since $A$ is hyperimmune-free, $g$ is dominated by a computable function $r$. Thus if we replace $W_{h(x)}$ by $W_{h(x), r(x)}$, we obtain a computable trace for $f$.

Lemma 4.9 and Proposition 4.14 together establish Theorem 3.1(II): if $A$ is Low $(\mathrm{CR}, \mathrm{SR})$, then $A$ is r.e. traceable by Theorem 3.1(I), and hyperimmunefree by Lemma 4.9. Thus by Proposition 4.14, $A$ is computably traceable.

As a corollary, we obtain an answer to the question of Ambos-Spies and Kučera.

Corollary 4.15. A real $A$ is $S$-low iff it is $S_{0}$-low.

Proof. This follows by Theorem 2.2 and Theorem 3.1(II), since each computably traceable real is $S_{0^{-}}$-low.

\section{Lowness notions RElated to Chaitin's halting PROBABility}

Recall that $A$ is array computable if there is a function $f \leq_{w t t} \emptyset^{\prime}$ bounding all functions computable from $A$ on almost all inputs.

Theorem 5.1. If $\Omega \in \mathrm{SR}^{A}$, then $A$ is array computable.

Proof. We show that the function $\beta(x)=\mu s \Omega_{s} \uparrow 3 x=\Omega \uparrow 3 x$ dominates each function $\alpha \leq_{T} A$. Since $\beta \leq_{w t t} \Omega \leq_{w t t} 0^{\prime}$, this shows that $A$ is array computable.

Given $\alpha \leq_{T} A$, consider the $A$-computable martingale $M=\sum_{p} M_{p}$, where $M_{p}$ is the martingale which has the value $2^{-p}$ on all strings of length up to $p$ and then doubles the capital along the string $y=\Omega_{\alpha(p)} \uparrow 3 p$, so that $M_{p}(y)=2^{p}$. Note that $M(z)$ is rational for each $z$. If $\alpha(p)>\beta(p)$ for infinitely many $p$, then $M$ Schnorr succeeds on $\Omega$, a contradiction.

Corollary 5.2. If $A$ is r.e., then $\Omega \in \mathrm{SR}^{A}$ iff $A$ is r.e. traceable.

Proof. For an r.e. set $A$, array computable implies r.e. traceable by the work of Ishmukhametov [5].

In [6] it is shown that r.e. traceable degrees do not contain diagonally noncomputable functions, and hence, by a result of Kučera 7], the r.e. traceable degrees have measure zero. On the other hand, every real $A$ which 
is Martin-Löf random relative to $\Omega$ satisfies that $\Omega$ is $\mathrm{MR}^{A}$, by van Lambalgen's theorem [15], and hence the measure of the set of $A$ such that $\Omega$ is $\mathrm{SR}^{A}$ is one; thus $A$ r.e. traceable is not equivalent to $\Omega \in \mathrm{SR}^{A}$. Also, $\Omega \in \mathrm{SR}^{A}$ is not equivalent to $A$ being array computable, as we now show.

The following notion of forcing appears implicitly in [4].

Definition 5.3. A tree $T$ is a set of strings $\sigma \in 2^{<\omega}$ such that if $\sigma \in T$ and $\tau$ is a substring of $\sigma$, then $\tau \in T$. A tree $T$ is full on a set $F \subseteq \omega$ if whenever $\sigma \in T$ and $|\sigma| \in F$, then $\sigma 0 \in T$ and $\sigma 1 \in T$. Let $F_{n}, n \in \omega$, be finite sets such that each $F_{n}$ is an interval of $\omega,\left|F_{n+1}\right|>\left|F_{n}\right|$, and $\bigcup_{n} F_{n}=\omega$. The sequence $F_{n}, n \in \omega$, is called a very strong array. Let $P$ be the set of computable perfect trees $T$ such that $T$ is full on $F_{n}$ for infinitely many $n$. Order $P$ by $T_{1} \leq_{P} T_{2}$ if $T_{1} \subseteq T_{2}$. The partial order $\left(P, \leq_{P}\right)$ is a notion of forcing that we call very strong array forcing.

Theorem 5.4. For each real $X$ there is a hyperimmune-free real $A$ such that no real computable from $X$ is in $\mathrm{SR}^{A}$. In particular, as hyperimmunefree implies array computable, there is an array computable real $A$ such that $\Omega \notin \mathrm{SR}^{A}$.

Proof. Let $A$ be sufficiently generic for very strong array forcing. Then $A$ is hyperimmune-free, as may be proved by modifying the standard construction of a hyperimmune-free degree [10] to work with trees that are full on infinitely many $F_{n}, n \in \omega$.

Moreover, for each real $B$ computable from $X$, there is an $n$ (hence infinitely many $n$ ) such that $A$ agrees with $B$ on $F_{n}$. Indeed, given a condition $T$, a condition extending $T$ and ensuring the existence of such an $n$ is obtained as a full subtree of $T$.

Hence no real $B$ computable from $X$ is Schnorr random relative to $A$. Indeed the measure of the set of those oracles $B$ that agree with $A$ on infinitely many $F_{n}$ is zero, and it is easy to see that the measure of those $B$ such that, for some $k>n, A$ and $B$ agree on $F_{k}$, goes to zero effectively as $n \rightarrow \infty$. Hence there is a Schnorr test relative to $A$ which is failed by any such $B$, as desired.

Question 5.5. Characterize the (r.e.) sets of integers $A$ such that $\Omega$ is computably random relative to $A$. Does this depend on the version of $\Omega$ used?

\section{REFERENCES}

[1] K. Ambos-Spies And A. KuČERA, Randomness in computability theory, in Computability Theory and Its Applications (Boulder, CO, 1999), Contemp. Math. 257, AMS, Providence, RI, 2000, pp. 1-14.

[2] B. Bedregal And A. Nies, Lowness properties of reals and hyper-immunity, in Proceedings of the 10th Workshop on Logic, Language, Information, and Computation, Ouro Preto, Minas Gerais, Brazil, 2003, Electron. Notes Theor. Comput. Sci. 84, Elsevier, 2003, online at http://www.elsevier.nl/locate/entcs/volume84.html. 
[3] A. Church, On the concept of a random sequence, Bull. Amer. Math. Soc., 46 (1940), pp. $130-135$.

[4] R. G. Downey, C. G. Jockusch, JR., And M. Stob, Array nonrecursive sets and genericity, in Computability, Enumerability, Unsolvability: Directions in Recursion Theory, S. B. Cooper, T. A. Slaman, and S. S. Wainer, eds., London Math. Soc. Lecture Note Ser., Cambridge University Press, Cambridge, UK, 1996, pp. 93-104.

[5] S. Ishmukhametov, Weak recursive degrees and a problem of Spector, Recursion Theory and Complexity (Kazan, 1997), de Gruyter Ser. Log. Appl. 2, de Gruyter, Berlin, 1999, pp. 81-87.

[6] B. Kuos-Hanssen, W. Merkle, and F. Stephan, Kolmogorov Complexity and the Recursion Theorem, to appear.

[7] A. KuČERA, Measure, $\Pi_{1}^{0}$-classes and complete extensions of PA, in Recursion Theory Week (Oberwolfach, 1984), Lecture Notes in Math. 1141, Springer, Berlin, 1985, pp. 245-259.

[8] A. KuČera And S. Terwijn, Lowness for the class of random sets, J. Symbolic Logic, 64 (1999), pp. 1396-1402.

[9] P. Martin-LöF, The definition of random sequences, Inform. and Control, 9 (1966), pp. 602-619.

[10] D. A. Martin And W. Miller, The degrees of hyperimmune sets, Z. Math. Logik Grundlag. Math., 14 (1968), pp. 159-166.

[11] A. NiEs, Lowness properties and randomness, Adv. Math., 197 (2005), pp. 274-305.

[12] A. Nies, F. Stephan, And S. Terwijn, Randomness, relativization, and Turing degrees, J. Symbolic Logic, 70 (2005), pp. 515-535.

[13] C.-P. SCHNORR, Zufälligkeit und Wahrscheinlichkeit. Eine algorithmische Begründung der Wahrscheinlichkeitstheorie, Lecture Notes in Math. 218, SpringerVerlag, Berlin, 1971.

[14] S. Terwijn And D. Zambella, Computational randomness and lowness, J. Symbolic Logic, 66 (2001), pp. 1199-1205.

[15] M. van Lambalgen, The axiomatization of randomness, J. Symbolic Logic, 55 (1990), pp. 1143-1167.

[16] D. Zambella, On Sequences with Simple Initial Segments, ILLC Technical Report ML 1990-05, University of Amsterdam, Amsterdam, The Netherlands, 1990. 